\title{
Rendimiento del frijol ayocote y maíz del agrosistema asociado en función del número de plantas por mata
}

\author{
Yield of ayocote bean and maize in an associated agrosystem in function \\ of the number of plants per hill
}

\author{
Néstor Jorge Rojas Victoria ${ }^{1 *}$, José Alberto Salvador Escalante Estrada ${ }^{1}$, \\ F. Víctor Conde Martínez ${ }^{1}$, José Apolinar Mejía Contreras² y Ramón Díaz Ruíz ${ }^{3}$
}

\footnotetext{
${ }^{1}$ Programa de Botánica, ${ }^{2}$ Programa de Producción de Semillas, Estrategias para el Desarrollo Agrícola Regional (PROEDAR), Colegio de Postgraduados, Campus Montecillo. Carretera México-Texcoco km 36.5. 56230 Montecillo, Texcoco, Estado de México, México.

‡Autor responsable (nerovic@colpos.mx)

${ }^{3}$ Colegio de Postgraduados, Campus Puebla. Carretera Federal México-Puebla km 125.5. 72760 Puebla, Puebla, México.
}

\section{RESUMEN}

El frijol ayocote (Phaseolus coccineus L.) es una leguminosa nativa de México con potencial alimenticio. Para lograr mayor distribución en el espacio, captación de radiación solar y producción de materia seca (MS), el ayocote trepador necesita un soporte. Los soportes convencionales como postes, mallas, rafia, elevan el costo de producción, sin embargo el ayocote puede sembrarse utilizando al maíz como soporte. Para incrementar el rendimiento de grano, se ha recurrido al manejo de la densidad de población y arreglo topológico. El objetivo del estudio conducido en Montecillo, México, en el ciclo primavera-verano de 2014, fue determinar si el número de plantas de frijol ayocote y maíz por mata en asociación, afectan la producción de biomasa, índice de cosecha, rendimiento de grano y sus componentes. Los tratamientos fueron nueve, resultado de la combinación de 1, 2 y 3 plantas de frijol ayocote y de 1,2 y 3 de maíz por mata. Se registraron las etapas fenológicas, biomasa total (BT), índice de cosecha (IC), rendimiento de grano (RG) y sus componentes en ambos cultivos. El diseño experimental fue bloques completos al azar con cuatro repeticiones. Los días a ocurrencia de las fases fenológicas en frijol ayocote y maíz, fueron similares entre tratamientos. En la asociación maíz-frijol los cambios en el número de plantas por mata de ambos cultivos, afectaron la BT, IC, RG y sus componentes tanto en ayocote como en maíz. En el agrosistema ayocote-maíz, la BT, RG y sus componentes, presentaron cambios por el número de plantas de ayocote y de maíz por mata. La combinación para lograr la mayor BT, RG e ingreso neto se logró con tres plantas de ayocote y una de maíz.

Cita recomendada:

Rojas Victoria, N. J., J. A. S. Escalante Estrada, F. V. Conde Martínez, J. A. Mejía Contreras y R. Díaz Ruíz. 2017. Rendimiento del frijol ayocote y maíz del agrosistema asociado en función del número de plantas por mata. Terra Latinoamericana 35: 219-228.
Palabras clave: densidad de población, arreglo topológico, biomasa, ingreso neto.

\section{SUMMARY}

The ayocote bean (Phaseolus coccineus L.) is a legume native to Mexico with potential as a foodstuff. To achieve greater spatial distribution to capture solar radiation and produce dry matter (MS), the climbing ayocote needs trellises. Conventional trellises raise the cost of production, but ayocote can be grown using maize as a trellis. To increase grain yield, management of population density and topological arrangement have been used. This study was conducted in Montecillo, Mexico, in the spring-summer season of 2014 to determine how the number of ayocote bean plants and maize per hill affects biomass production, harvest index, and grain yield and its components. The nine treatments were the result of the combination of 1, 2 and 3 ayocote bean plants and 1,2 and 3 maize plants per hill. We recorded days to each phenological stage, total biomass (TB), harvest index $(\mathrm{HI})$, grain yield (GY) and yield components of both crops. The experimental design was randomized complete blocks with four repetitions. The duration of the phenological phases of ayocote bean and maize were similar among treatments. In the association maize-ayocote the changes in the number of plants per hill of both crops affected TB, HI, GY and yield components of both ayocote bean and maize. In the agrosystem ayocotemaize, TB, GY and yield components are affected by the number of plants of bean ayocote and maize per hill. The combination that achieved the highest TB, GY and net income was three ayocote plants and one maize plant.

Recibido: enero de 2017. Aceptado: junio de 2017. Publicado en Terra Latinoamericana 35: 219-228. 
Index words: population density, topological arrangement, biomass, net income.

\section{INTRODUCCIÓN}

El frijol ayocote (Phaseolus coccineus L.) es una leguminosa nativa de México, originaria de las zonas templadas, con gran potencial y principalmente uso alimenticio, además del aprovechamiento de todas sus partes flores, vainas, semillas y follaje (Ayala et al., 2006) En la actualidad, la especie representa una opción de producción de alimentos en áreas marginales en Valles altos de México. El rendimiento en grano (RG) de un cultivo depende de varios factores, como son: variación en tipo de suelo, elementos del clima, cultivar utilizado y manejo de cultivo (Escalante et al., 2013), dentro de lo cual la densidad de población (DP) puede ser determinante para el incremento del $\mathrm{RG}$, que puede estar en función de la duración del ciclo de crecimiento, hábito de crecimiento del cultivar, fertilización y disponibilidad de agua, entre otros factores.

El frijol ayocote existen cultivares de tipo trepador, por lo cual es necesario el uso de espalderas, como la convencional que consiste en postes de madera común o de estructuras metálicas con malla, que elevan considerablemente el costo de producción, algunos agricultores no utilizan tutores en la siembra del ayocote y el rendimiento es bajo. Una alternativa es la siembra de frijol ayocote en asociación con maíz, el cual funciona como tutor o espaldera viva. Dicha asociación presenta ventajas, ya que ambas especies por sus diferencias morfológicas y fisiológicas se complementan mutuamente en el uso de los recursos (agua, radiación, nutrimentos), de la misma forma, influye en la distribución en el espacio, captación de radiación solar y en consecuencia mayor producción de dosel (Delgado et al., 2014; Rojas et al., 2015). Sin embargo, cuando se siembra bajo este arreglo existe una reducción en el rendimiento de una o ambas especies, debido a la competencia interespecífica. La leguminosa es la más afectada bajo este esquema con una reducción de 25 a $50 \%$ en relación al monocultivo (Vélez et al., 2007; Escalante et al., 2015). Por otra parte, se asume que, bajo condiciones no limitantes de agua y nutrimentos, la reducción en el RG en frijol se debe al sombreado ocasionado por el maíz, que puede limitar una mayor expresión del número de granos y consecuentemente el RG (Fageria et al., 2010; Delgado et al., 2015; Rojas et al., 2015). Para incrementar dicho RG se ha recurrido a diferentes prácticas agrícolas, densidad de población (DP) y arreglo topológico (AT) (Jiménez y Acosta, 2013). Los cambios en la DP se pueden lograr al elevar el número de plantas por hilera o el número de plantas por mata en cada hilera para buscar mayor intercepción de la radiación fotosintéticamente activa (RFA), un uso más eficiente del agua y nutrimentos (Escalante y Rodríguez, 2010).

Algunos estudios señalan que el AT y el aumento en DP no afectan los días a ocurrencia de las fases fenológicas en frijol, aunque independiente de la arquitectura del cultivar, disminuye la acumulación de biomasa (BT) y RG por planta, pero no afecta la distribución de materia seca en los órganos de la planta. Sin embargo, la producción de BT y el RG por unidad de superficie se incrementa hasta un $20 \%$ con respecto al testigo (Delgado et al., 2015). Escalante et al. (2014); mencionan que a mayor DP, el RG por hectárea y la altura de la planta se incrementan. En la medida en que se incrementa el número de plantas, aumenta la cobertura vegetal por área del cultivo; reduciendo la pérdida de agua por evaporación al cubrir mayor área del suelo y aumentando la captación de radiación solar, como consecuencia, se tiene una mayor producción de MS (Osuna et al., 2012; Jiménez y Acosta, 2013). Delgado et al. (2014) al evaluar el efecto de la DP y nitrógeno sobre el rendimiento del frijol ejotero en espaldera viva de maíz, en Montecillo, Estado de México, encontraron que el rendimiento de ejote y número de ejotes más alto ocurrió con dos plantas de frijol por mata y $150 \mathrm{~kg}$ de $\mathrm{N} \mathrm{ha}^{-1}$. Por otra parte, los resultados de Apáez et al. (2013) en frijol chino asociado con maíz y girasol en Montecillo, Estado de México, reportan que el RG y BT de frijol está determinado por el cultivo utilizado como tutor. El objetivo del estudio fue determinar si el número de plantas de maíz y frijol ayocote por mata en la asociación de estos cultivos afectan la producción de biomasa, rendimiento y sus componentes de grano del frijol ayocote, maíz en unicultivo y del agrosistema ayocote-maíz.

\section{MATERIALES Y MÉTODOS}

El estudio se estableció en el campus experimental del Colegio de Postgraduados, Montecillo, Estado de México, (19²9’ N y $98^{\circ} 54^{\prime} \mathrm{O}, 2250 \mathrm{~m}$ de altitud), con 
clima $\mathrm{Cw}$ que corresponde al templado (García, 2005), con período libre de heladas de 170 días, temperatura media anual de $15.4{ }^{\circ} \mathrm{C}$ y precipitación media anual de $512 \mathrm{~mm}$; el suelo es de textura franco-arenosa, con $\mathrm{pH}$ de 7.1, CE de $0.49 \mathrm{dSm}^{-1}$ (sin problemas de salinidad), presentó un contenido de materia orgánica (MO) de 2.3\%; con $26.25 \mathrm{~kg}$ de $\mathrm{N}$ aprovechable, amonio (25.9 $\left.\mathrm{mg} \mathrm{kg}^{-1}\right)$, el cultivo se desarrolló solo con la fertilidad residual del suelo. La siembra se realizó el 5 de mayo de 2014, con un cultivar (Cv) de frijol ayocote (A) procedente de Juchitepec, Estado de México, México. El grano es de color violeta, hábito de crecimiento indeterminado trepador (Tipo IV); y maíz nativo azul (M) de la raza chalqueño. La distancia entre matas fue de $0.30 \mathrm{~m}$ y $0.80 \mathrm{~m}$ entre hileras. La distribución y el arreglo de tratamientos se muestra en el Cuadro 1. El diseño experimental fue bloques al azar con cuatro repeticiones. La unidad experimental consistió de tres surcos de $5 \mathrm{~m}$ de longitud con orientación este a oeste, tomando el surco central como parcela útil (menos $50 \mathrm{~cm}$ de cada extremo).

Durante el desarrollo del cultivo se registraron variables climáticas: temperatura máxima (Tmáx) y mínima (Tmín) diaria, evaporación (Ev) (por medio de la tina de evaporación), y precipitación (PPT) ( datos proporcionados por la estación agrometeorológica del Colegio de Postgraduados, Campus Montecillo); además los días a ocurrencia de las fases fenológicas como: emergencia (E), Etapa vegetativa (EV), floración (R6) y madurez fisiológica (R9) para frijol ayocote, bajo los criterios presentados en Escalante y Kohashi (2015), mientras que para maíz, los días a:

Cuadro 1. Tratamientos en la siembra asociada frijol ayocotemaíz, Montecillo, México. Verano 2014.

\begin{tabular}{lccc}
\hline Tratamiento & $\begin{array}{c}\text { Plantas de frijol } \\
\text { ayocote }\end{array}$ & Plantas de maíz & Simbología \\
\hline 1 & 1 & 1 & $1 \mathrm{~A}+1 \mathrm{M}$ \\
2 & 1 & 2 & $1 \mathrm{~A}+2 \mathrm{M}$ \\
3 & 1 & 3 & $1 \mathrm{~A}+3 \mathrm{M}$ \\
4 & 2 & 1 & $2 \mathrm{~A}+1 \mathrm{M}$ \\
5 & 2 & 2 & $2 \mathrm{~A}+2 \mathrm{M}$ \\
6 & 2 & 3 & $2 \mathrm{~A}+3 \mathrm{M}$ \\
7 & 3 & 1 & $3 \mathrm{~A}+1 \mathrm{M}$ \\
8 & 3 & 2 & $3 \mathrm{~A}+2 \mathrm{M}$ \\
9 & 3 & 3 & $3 \mathrm{~A}+3 \mathrm{M}$ \\
\hline A = frijol ayocote; $\mathrm{M}=$ maíz. $4.16,8.3$ y 12.3 plantas $\mathrm{m}^{-2}$ para cada especie.
\end{tabular}

emergencia (E), etapa vegetativa (EV), floración (R1) y madurez fisiológica (R6) según el criterio presentado en Hanway (1963), el registro de las etapas fue cuando el $80 \%$ de las plantas presentaron las características antes mencionadas. A la cosecha del frijol ayocote se registró el rendimiento de grano $\left(\mathrm{RG}, \mathrm{g} \mathrm{m}^{-2}\right)$, y sus componentes como: número de vainas con grano $\mathrm{m}^{-2}$ $(\mathrm{NV})$; número de granos por vaina $(\mathrm{GV})$; el tamaño del grano (g) mediante el peso de 100 granos (PCG); número de granos $\mathrm{m}^{-2}(\mathrm{NG})$, biomasa total (materia seca, $\mathrm{g} \mathrm{m}^{-2}, \mathrm{BT}$ ) (sin incluir raíz); índice de cosecha (IC) calculado mediante la relación: $\mathrm{IC}=\mathrm{RG} / \mathrm{BT}$. En maíz se evaluó el rendimiento de grano ( $\mathrm{RG}, 10 \%$ de humedad, $\mathrm{g} \mathrm{m}^{-2}$ ); número de hileras $(\mathrm{NH})$; número de granos por hilera (NGH); longitud de mazorca (LM); diámetro de mazorca (DM) (parte central), el tamaño del grano (g) mediante el peso de 100 granos (PCG), número de granos $\mathrm{m}^{-2}(\mathrm{NG})$; biomasa total $\left(\mathrm{MS} \mathrm{g} \mathrm{m}^{-2}\right.$, BT) ( sin incluir raíz), índice de cosecha (IC) con la relación $\mathrm{IC}=(\mathrm{RG} / \mathrm{BT}) * 100$. A lo datos de las variables en estudio se les aplicó un análisis de varianza y la prueba de comparación de medias de Tukey $\alpha=0.05$, utilizando el programa SAS versión 9.2 (SAS 2003). Adicionalmente, se realizó un análisis económico a la variable de rendimiento de grano para frijol ayocote y grano en maíz para determinar el mayor ingreso neto del agrosistema, utilizando la siguiente relación (Volke, 1982):

$\mathrm{IN}=\mathrm{Y}^{*} \mathrm{Py}-\left(\Sigma \mathrm{X}_{\mathrm{i}} \mathrm{P}_{\mathrm{i}}+\mathrm{P}_{2}\right)$

Dónde:

$\mathrm{IN}=$ ingreso neto,

$\mathrm{Y}=$ rendimiento $\left(\mathrm{kg} \cdot \mathrm{ha}^{-1}\right)$,

Py $=$ precio del grano $\left(\right.$ pesos $\left.\cdot \mathrm{kg}^{-1}\right)$,

$\Sigma \mathrm{X}_{\mathrm{i}} \mathrm{P}_{\mathrm{i}}=$ suma de costos variables $\left(\right.$ pesos $\left.\cdot \mathrm{ha}^{-1}\right)$,

$\mathrm{P}_{2}=$ costos fijos (pesos $\cdot \mathrm{ha}^{-1}$ ).

Costo fijo (CF): incluye manejo del cultivo, manejo de plagas y enfermedades, manejo de maleza, mano de obra, transporte, cosecha.

Costo variable $(\mathrm{CV})$ : precio y cantidad de semilla y fertilizante

Costo total (CT): Costo fijo + costo variable Ingreso total (IT): rendimiento por el precio de $\mathrm{kg}$ de grano de frijol ayocote $(\$ 25.00)$ y maíz $(\$ 6.00)$

Ingreso neto (IN): Ingreso total - costo total

Tasa de retorno (TR): IN/CT 


\section{RESULTADOS Y DISCUSIÓN}

\section{Fenología y Elementos del Clima}

Para frijol ayocote y maíz, los tratamientos impuestos (número de plantas por mata) no afectaron los días a ocurrencia de las fases fenológicas presentándose similitudes entre tratamientos. En frijol ayocote las fases de E, R6 y R9 ocurrieron a los 12, 70 y 126 días después de la siembra (dds), en todos los casos, respectivamente. Para maíz, la E, R1 y R6 se presentaron a los 10, 69 y $148 \mathrm{dds}$, de igual manera en todos los casos, respectivamente. Durante el desarrollo del cultivo, la precipitación (PP) estacional fue de $412 \mathrm{~mm}$, de la cual el 56\% ocurrió en la etapa vegetativa y el 44\% restante en la reproductiva (Figura 1).

Resultados semejantes han sido reportados en frijol común de hábito de crecimiento indeterminado en asociación con girasol y maíz para la región de Texcoco, México (Salinas et al., 2008; Díaz et al., 2010; Delgado et al., 2014). Por otra parte, se observó que, durante las etapas vegetativa y reproductiva para ambos cultivos, la temperatura máxima y mínima promedio fue de 30 ${ }^{\circ} \mathrm{C}$ y $5{ }^{\circ} \mathrm{C}$. La temperatura máxima fue superior a la óptima para frijol ayocote que se reporta en un intervalo de 25 a $28^{\circ} \mathrm{C}$ (Masaya y White, 1991; Barrios-Gómez et al., 2010). La temperatura más alta en promedio fue de $35^{\circ} \mathrm{C}$. Ritchie y NeSmith (1991) señalan que la temperatura entre los 25 a $28{ }^{\circ} \mathrm{C}$ favorece el desarrollo de un mayor dosel vegetal de frijol ayocote. Asimismo, la precipitación y humedad residual influyen en el desarrollo del cultivo, específicamente su variación en las primeras etapas de crecimiento (Escalante, 2001; Barrios-Gómez et al., 2011).

\section{Biomasa Total (BT), Índice de Cosecha (IC\%) y Rendimiento de Grano (RG) de Frijol Ayocote}

La (BT), (IC) y (RG) de frijol ayocote mostraron cambios significativos por efecto del NPA y NPM por mata (Cuadro 2). Los valores más altos de BT se registraron con los tratamientos $3 \mathrm{~A}+1 \mathrm{M}$ y $3 \mathrm{~A}+2 \mathrm{M}$ con $859\left(8590 \mathrm{~kg} \mathrm{ha}^{-1}\right)$ y $839 \mathrm{~g} \mathrm{~m}^{-2}\left(8390 \mathrm{~kg} \mathrm{ha}^{-1}\right)$, respectivamente; los cuales superaron al resto de los tratamientos en $30 \%$. Los tratamientos $1 \mathrm{~A}+1 \mathrm{M}$, $1 \mathrm{~A}+2 \mathrm{M}$ y $1 \mathrm{~A}+3 \mathrm{M}$ presentaron los valores más bajos de estas variables. Tendencias semejantes han sido reportadas por Vélez et al. (2007) y Díaz et al. (2010). Delgado et al. (2015) al estudiar el efecto de la DP sobre el rendimiento del frijol ejotero en espaldera

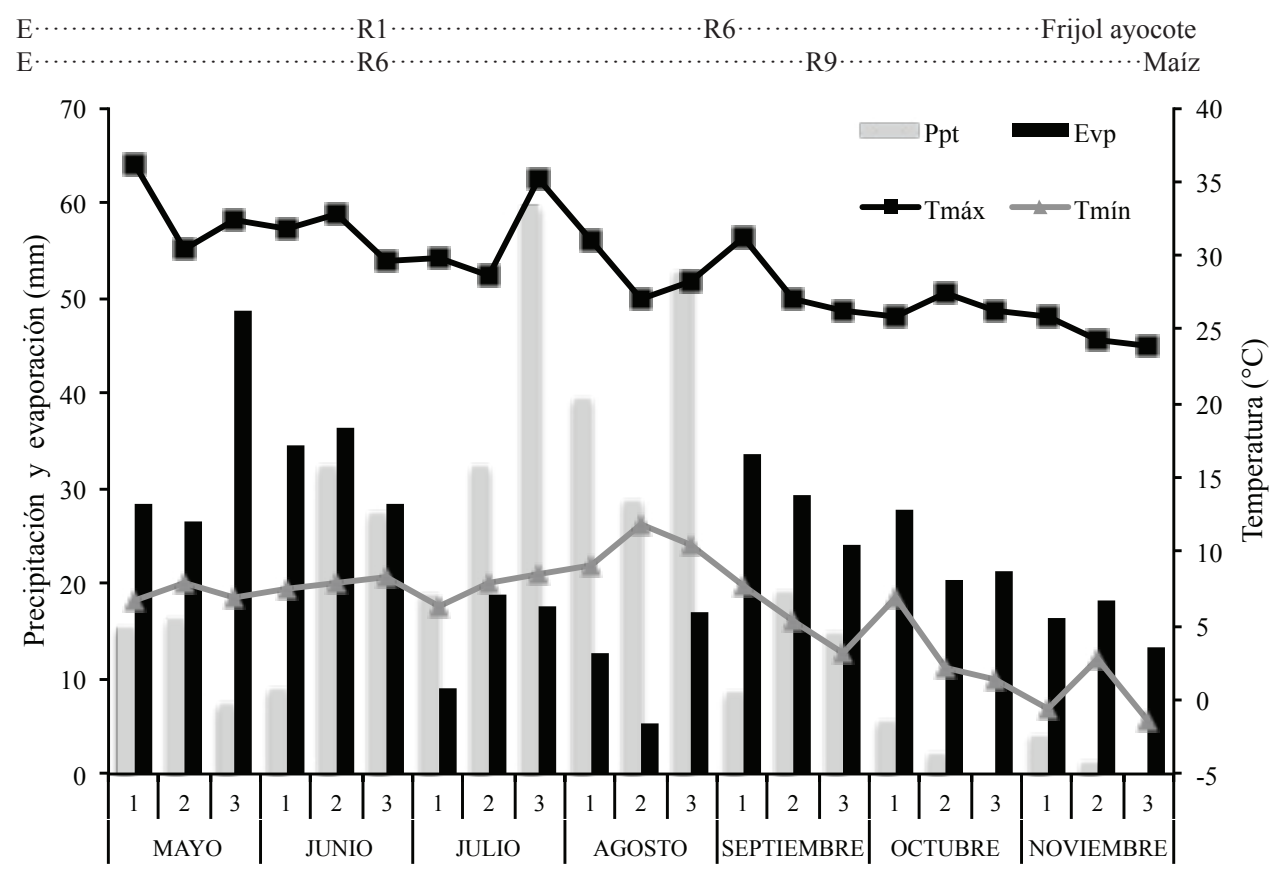

Figura 1. Temperatura máxima (Tmáx) y mínima (Tmín) (media decenal), la suma decenal de la evaporación ( $\mathrm{Ev}, \mathrm{mm})$ y precipitación $(\mathrm{Ppt}, \mathrm{mm})$ durante el ciclo de los cultivos de maíz y frijol ayocote. Montecillo, Méx. 2014. Para frijol ayocote $\mathbf{E}=$ emergencia, $\mathbf{E v}=$ etapa vegetativa, R6 = floración y R9 = madurez fisiológica; para maíz R1 = floración y R6 = madurez fisiológica. 
Cuadro 2. Biomasa, índice de cosecha, rendimiento de grano y sus componentes de frijol ayocote ( $P$. coccineus L.) en asociación, en función del NPA y NPM por mata. Montecillo, México. Verano-Otoño 2014.

\begin{tabular}{|c|c|c|c|c|c|c|c|c|}
\hline Tratamiento & RG & RG & BT & IC & $\mathrm{TG}$ & NG & NV & GV \\
\hline & $\mathrm{kg} \mathrm{ha}^{-1}$ & $-\cdots \mathrm{gm}^{2}-$ & $-\cdots$ & $\%$ & $\mathrm{~g}$ & $-\cdots$ & $\ldots$ & \\
\hline $3 \mathrm{~A}+1 \mathrm{M}$ & 2580 & $258 \mathrm{a}$ & $860 \mathrm{a}$ & $30 \mathrm{~b}$ & $0.27 \mathrm{~b}$ & $945 \mathrm{a}$ & $568 \mathrm{a}$ & $3.4 \mathrm{c}$ \\
\hline $3 \mathrm{~A}+2 \mathrm{M}$ & 2500 & $250 \mathrm{ab}$ & $839 a$ & $30 b$ & $0.25 b$ & $975 a$ & $520 \mathrm{a}$ & $3.4 \mathrm{c}$ \\
\hline $3 \mathrm{~A}+3 \mathrm{M}$ & 2360 & $236 b$ & $814 a$ & $29 \mathrm{c}$ & $0.24 b$ & $970 \mathrm{a}$ & $523 a$ & $3.1 \mathrm{~d}$ \\
\hline $2 \mathrm{~A}+2 \mathrm{M}$ & 1980 & $189 \mathrm{c}$ & $615 b$ & $31 \mathrm{a}$ & $0.26 \mathrm{~b}$ & $709 b$ & $408 \mathrm{c}$ & $3.3 \mathrm{c}$ \\
\hline $2 \mathrm{~A}+1 \mathrm{M}$ & 1790 & $179 \mathrm{c}$ & $606 b$ & $29 \mathrm{c}$ & $0.28 b$ & $645 b$ & $451 \mathrm{c}$ & $3.5 b$ \\
\hline $2 \mathrm{~A}+3 \mathrm{M}$ & 1730 & $173 d$ & $586 b$ & $29 \mathrm{c}$ & $0.26 \mathrm{~b}$ & $653 b$ & $349 d$ & $3.1 \mathrm{~d}$ \\
\hline $1 \mathrm{~A}+1 \mathrm{M}$ & 1190 & $119 \mathrm{e}$ & $421 \mathrm{c}$ & $28 \mathrm{c}$ & $0.30 \mathrm{a}$ & $391 \mathrm{c}$ & $317 \mathrm{e}$ & $3.8 \mathrm{a}$ \\
\hline $1 \mathrm{~A}+2 \mathrm{M}$ & 1130 & $113 \mathrm{e}$ & $410 \mathrm{~d}$ & $27 d$ & $0.29 \mathrm{a}$ & $383 c$ & $300 \mathrm{e}$ & $3.6 b$ \\
\hline $1 \mathrm{~A}+3 \mathrm{M}$ & 1080 & $108 \mathrm{e}$ & $400 \mathrm{~d}$ & $27 \mathrm{~d}$ & $0.31 \mathrm{a}$ & $344 \mathrm{c}$ & $307 \mathrm{e}$ & $3.8 \mathrm{a}$ \\
\hline Media general & 1810 & 181 & 617 & 29 & 0.28 & 668 & 416 & 3.4 \\
\hline Tukey 5\% & 210 & 21 & 174 & 11 & 0.065 & 466 & 170 & 0.19 \\
\hline Prof. F: $* *<0.01$ & $* *$ & $* *$ & $* *$ & $* *$ & $* *$ & $* *$ & $* *$ & $* *$ \\
\hline
\end{tabular}

$\mathrm{A}=$ frijol ayocote, $\mathrm{M}=$ maíz; el número indica el número de plantas por mata. $\mathrm{BT}=$ biomasa total; $\mathrm{IC}=$ índice de cosecha; $\mathrm{RG}=$ rendimiento de grano; $\mathrm{TG}=$ tamaño del grano (g); $\mathrm{NG}=$ número de granos; $\mathrm{NV}=$ número de vainas; $\mathrm{GV}=$ granos por vaina; $\mathrm{NPA}=$ número de plantas de frijol ayocote, $\mathrm{NPM}=$ número de plantas de maíz. Letras distintas en la misma columna indican diferencias significativas, según (Tukey $\alpha=0.05$ ), ${ }^{* *} P<0.01$.

viva de maíz, encontraron que el mayor rendimiento y número de ejotes se logró con dos plantas de frijol y una de maíz, en el presente estudio se incrementó el RG al aumentar el número de plantas de frijol ayocote (tres plantas).
Por otra parte, para maíz los valores más altos de BT se lograron con $3 \mathrm{M}+1 \mathrm{~A}$, seguido por los tratamientos $3 \mathrm{M}+2 \mathrm{~A}$ y $3 \mathrm{M}+3 \mathrm{~A}\left(1662,1510\right.$ y $1417 \mathrm{~g} \mathrm{~m}^{-2},(16620$, 15 100, y $14170 \mathrm{~kg} \mathrm{ha}^{-1}$ ), respectivamente) (Cuadro 3). Los valores más bajos fueron con el tratamiento $1 \mathrm{M}+3 \mathrm{~A}$

Cuadro 3. Biomasa, índice de cosecha, rendimiento de grano y sus componentes de maíz chalqueño cv azul, en asociación con frijol ayocote. Montecillo México. Verano-Otoño. 2014.

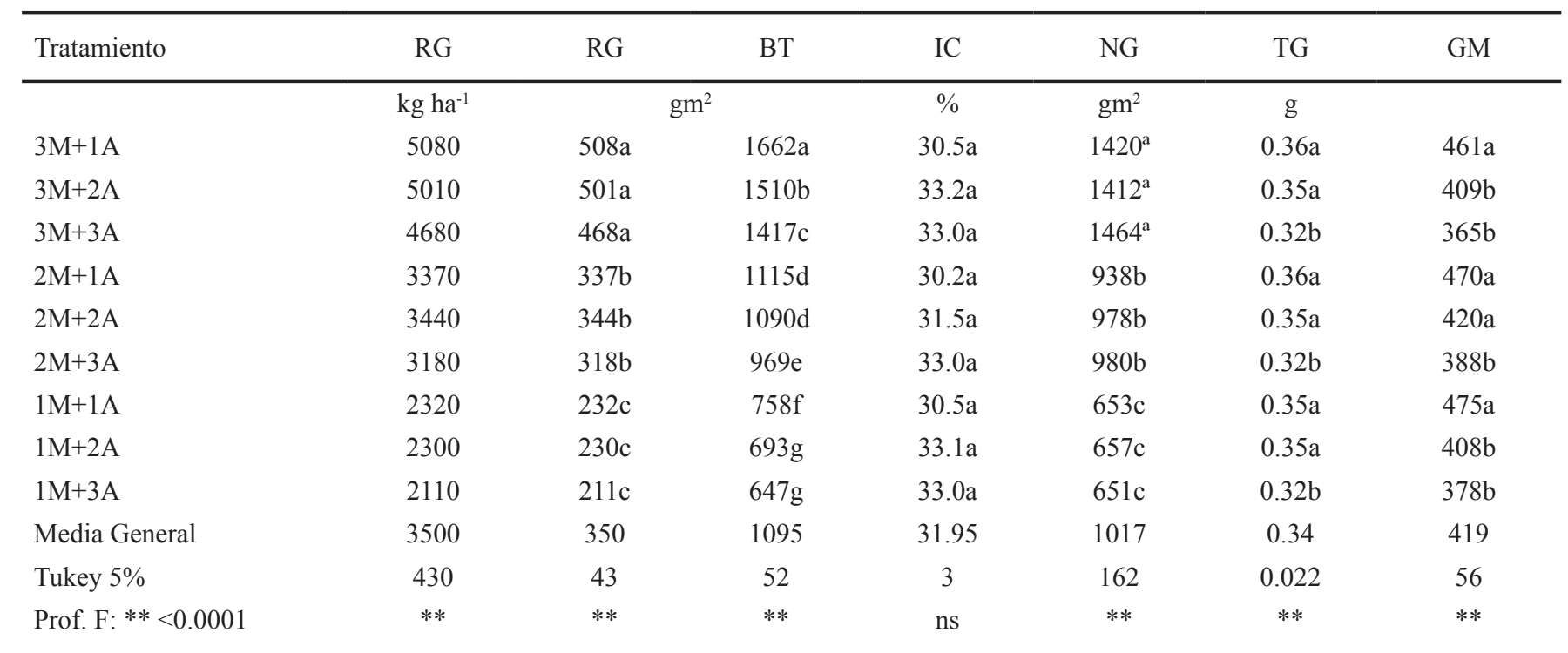

$\mathrm{A}=$ frijol ayocote, $\mathrm{M}$ = maíz; el número indica el número de plantas. $\mathrm{BT}=$ biomasa total; $\mathrm{IC}=$ índice de cosecha; $\mathrm{RG}=$ rendimiento de grano; $\mathrm{TG}=$ tamaño del grano $(\mathrm{g}) ; \mathrm{NG}=$ número de granos; $\mathrm{GM}=$ granos por mazorca; $\mathrm{MG}=$ media general; letras distintas en la misma columna indican diferencias significativas, según (Tukey $\alpha=0.05$ ), ${ }^{* *} P<0.01$; indican efecto significativo. 
que presentó $647 \mathrm{~g} \mathrm{~m}^{-2}$. Esta diferencia en BT entre tratamientos se puede atribuir a la capacidad del maíz para captar la energía solar (Escalante et al., 2006), puesto que al incrementar DP, se aumenta el índice de área foliar; con esto la cantidad de radiación interceptada y en consecuencia la producción de BT, así como la competencia por radiación y recursos generada por el número de plantas de maíz (Escalante et al., 2006; Garduño et al., 2009).

\section{Índice de Cosecha (IC)}

Para el IC de frijol ayocote se encontraron diferencias significativas por efecto de los tratamientos. Esto indica que los cambios en el NPA y NPM afectaron la distribución de MS hacia el grano de frijol ayocote (Cuadro 2). La producción del tratamiento de $2 \mathrm{~A}+2 \mathrm{M}$ mostró los valores más altos del índice de cosecha seguido por $3 \mathrm{~A}+2 \mathrm{M}$ (31 y $30 \%$, respectivamente). El IC promedio fue de $29 \%$. En estudios recientes indican que el rendimiento de grano y el índice de cosecha de frijol, se redujeron significativamente cuando fueron sembrados simultáneamente con maíz, debido principalmente a la competencia por insumos generada por esta especie (Santalla et al., 1999; Apáez et al., 2013). En cuanto al IC en maíz Azul no hubo diferencias significativas entre los tratamientos (Cuadro 3). Los cambios en el número de plantas de frijol ayocote y maíz no afectaron la acumulación de MS en las estructuras de las plantas ni su distribución hacia el grano. Lo anterior se puede atribuir a que el maíz es más competitivo que el frijol, el cual desarrolla un dosel más amplio al del frijol ayocote logrando mayor captación de radiación y producción de fotoasimilados, generando mayor acumulación de MS, asimismo, los fotoasimilados y MS son exportados a los órganos de interés económico en este caso a la formación y llenado de grano, esto coincide con lo señalado por Vélez et al. (2007), quienes evaluaron los efectos de competencia en el cultivo asociado maíz-frijol voluble trepador.

\section{Rendimiento de Grano y sus Componentes}

En frijol ayocote presentó cambios significativos entre tratamientos en las variables del rendimiento de grano (RG), número de granos $(\mathrm{NG})$ y número de vainas (NV) (Cuadro 2). Con 3A+1M y 3A+2M se logró el $\mathrm{RG}$ de frijol ayocote más alto con $258\left(2580 \mathrm{~kg} \mathrm{ha}^{-1}\right) \mathrm{y}$ $250 \mathrm{~g} \mathrm{~m}^{-2}$ (2500 kg ha ${ }^{-1}$ ), respectivamente (Cuadro 2).
En NG y el NV presentó tendencias similares. Los valores más bajos de $\mathrm{RG}$, NG y $\mathrm{NV}$ se obtuvieron con los tratamientos $1 \mathrm{~A}+1 \mathrm{M}, 1 \mathrm{~A}+2 \mathrm{M}$ y $1 \mathrm{~A}+3 \mathrm{M}$. En contraste, los valores del tamaño de grano (TG) y granos por vaina $(\mathrm{GV})$ fueron similares entre tratamientos (Cuadro 2). Esto significa que el aumento en $\mathrm{RG}$ fue producto de un aumento en $\mathrm{NG}$ y $\mathrm{NV}$ en los tratamientos donde se tuvo mayor NPA (Cuadro 2). Delgado et al. (2015) evaluaron el efecto de 4, 8 y 12 plantas $\mathrm{m}^{-2}$ sobre el rendimiento y rentabilidad económica del frijol ejotero en espaldera viva de maíz. El rendimiento y número de ejotes más alto se encontró con 8 plantas $\mathrm{m}^{-2}$ de frijol por mata, coincidiendo con lo reportado por Jiménez y Acosta (2013) quienes indican que para la variedad Pinto Saltillo de grano, la densidad promedio recomendada es de 8 a 16 plantas $\mathrm{m}^{-1}$ lineal. Osuna et al. (2012) quienes trabajaron con frijol Pinto en altas DP bajo temporal obtuvieron rendimientos altos con densidades de 145 y 260 mil plantas ha ${ }^{-1}$ (14 y 26 plantas $\mathrm{m}^{-2}$, respectivamente). Por otra parte, Alves et al. (2008), al aumentar la densidad de 100 mil a 500 mil plantas ha ${ }^{-1}$ (10 y 50 plantas $\mathrm{m}^{-2}$, respectivamente) en cultivares de frijol bajo temporal, encontraron que el rendimiento de grano se incrementó de $1962 \mathrm{~kg} \mathrm{ha}^{-1} \mathrm{a}$ $3410 \mathrm{~kg} \mathrm{ha}^{-1}$; también reportan reducción en el número de granos por vaina y el número de vainas por planta al incrementar la densidad de plantas de frijol. Tsubo y Walker (2004), señalan que el maíz ejerce sobre el frijol de tipo arbustivo una fuerte competencia, que puede reducir hasta un $67 \%$ la producción de BT y en $40 \%$ la MS en la vaina. Clark y Francis (1985), al evaluar diferentes cultivares de frijol de hábito de crecimiento determinado e indeterminado asociados con maíz, observaron reducciones de 25 y $8 \%$ en el número de nudos, 46 y $39 \%$ en el número de racimos, y 53 y $44 \%$ en el número de vainas, respectivamente, con relación a sus unicultivos. En contraste, Tsubo et al. (2003) no encontraron reducciones significativas en la BT y RG en cultivares de frijol de crecimiento indeterminado cuando fueron asociados con maíz con densidades de 2.2, 4.4, 6.7 y 12.5 plantas $\mathrm{m}^{-2}$, lo anterior debido al menor sombreado del maíz sobre los cultivares de frijol (Díaz et al., 2010) quienes trabajaron con densidades de 6.2 y 12.4 plantas $\mathrm{m}^{-2}$ de frijol asociado con girasol.

En maíz, el RG, NG y GM mostraron diferencias significativas por efecto de los tratamientos (Cuadro 3). Con $3 \mathrm{M}+1 \mathrm{~A}$ y $3 \mathrm{M}+2 \mathrm{~A}$ se logró el $\mathrm{RG}$ de maíz más alto con $508 \mathrm{~g} \mathrm{~m}^{-2}$ y $501 \mathrm{~g} \mathrm{~m}^{-2} 5080$ y $5010 \mathrm{~kg} \mathrm{ha}^{-1}$ ), respectivamente (Cuadro 3). En las variables de NG y 
GM presentaron una tendencia similar. Esto se puede atribuir que a mayor NPM y menor NPA se puede incrementar el RG, NG y GM del maíz. En contraste, el TG no mostró cambios significativos por efecto del NPM. Esto indica que el maíz presenta una notable respuesta al aumento en la DP en términos de producción de BT como resultado de una mayor captura de luz por el cultivo. Jana et al. (2000) estudiaron la eficiencia de la asociación maíz-frijol común y encontraron un RG $\left(25 \mathrm{~g} \mathrm{~m}^{-2}\right)$ más bajo en la asociación que en unicultivo $\left(76 \mathrm{~g} \mathrm{~m}^{-2}\right)$, esto sugiere diferencias en el grado de competencia de las especies utilizadas como espaldera viva. Por su parte, Galdámez et al. (2010) estimaron la producción de BT y RG de maíz asociado con frijol, canavalia y calabaza, el mayor RG se logró con maíz en unicultivo (7.13 $\mathrm{Mg} \mathrm{ha}^{-1}$ ) seguido por la asociación maíz-frijol $\left(6.70 \mathrm{Mg} \mathrm{ha}^{-1}\right)$ y el más bajo (3.57 $\left.\mathrm{Mg} \mathrm{ha}^{-1}\right)$ con el sistema maíz-calabaza-canavalia. En contraste, Morgado y Willey (2008) evaluaron el efecto de DP de plantas de maíz y frijol en cultivos intercalados, obtuvieron un RG en maíz de $6195 \mathrm{~kg} \mathrm{ha}^{-1}$ con 40000 plantas ha-1 de maíz y $4941 \mathrm{~kg} \mathrm{ha}^{-1}$ con 60000 plantas ha $^{-1}$ de frijol. Asimismo, Mutungamiri et al. (2001), indican que en la asociación maíz-frijol, hay una reducción del 69\% en el RG del maíz al bajar la DP de 37000 a 24000 plantas ha $^{-1}$, pero hay aumento del 37\% en el RG de frijol.

En frijol ayocote, el RG presentó una alta relación con el NG $\left(\mathrm{R}^{2}=0.93^{*}\right)$ y NV $\left(\mathrm{R}^{2}=0.96^{* *}\right)$ (Cuadro 4). Esto sugiere que para lograr incrementos en el RG del frijol ayocote, se tiene que incrementar la DP la cual aumentará el tamaño del dosel y la producción de MS, proporcionando los fotosintatos necesarios para formar un mayor NV y NG (Escalante y Rodríguez, 2011). Por otra parte, el maíz presentó tendencias similares, el RG mostró una alta relación con el $\mathrm{NG}\left(\mathrm{R}^{2}=0.98^{* *}\right)$,

Cuadro 4. Correlación entre el rendimiento de grano con el número de granos (NG), número de vainas (NV), número de granos por vaina (GV) y tamaño de grano (TG).

\begin{tabular}{lcc}
\hline $\begin{array}{l}\text { Componentes } \\
\text { de rendimiento }\end{array}$ & $\begin{array}{c}\text { Coeficiente de } \\
\text { determinación de } \mathrm{R}^{2}\end{array}$ & Probabilidad de F \\
\hline $\mathrm{NG}$ & 0.93 & $* *$ \\
$\mathrm{NV}$ & 0.96 & $* *$ \\
$\mathrm{GV}$ & 0.60 & $\mathrm{NS}$ \\
$\mathrm{TG}$ & 0.80 & $\mathrm{NS}$ \\
\hline
\end{tabular}

$\mathrm{NS}=$ no significativo $(P \leq 0.05) ; * * 0.01(P \leq 0.01)$.
(Cuadro 5). Lo anterior indica, que el maíz al tener un dosel amplio puede captar mayor radiación solar y producir mayor cantidad de MS, la cual se destina al llenado de grano (Escalante et al., 2015).

Los resultados muestran que la reducción del rendimiento del maíz y del ayocote por efecto de NPM y NPA se puede atribuir a la competencia ejercida por el maíz hacia el ayocote, dicha competencia puede reducir la producción de biomasa total hasta un $40 \%$ lo que puede afectar el llenado de grano y el RG, lo anterior se puede atribuir a que el maíz es más competitivo que el ayocote, puesto que desarrolla un dosel más amplio y en consecuencia mayor captación de radiación, producción de fotoasimilados y acumulación de materia seca, que son exportados a los órganos de interés económico, en este caso a la formación y llenado de grano.

Esto coincide con lo reportado por Morales et al. (2008) señalan que en el frijol sembrado en asociado, la producción de biomasa y rendimiento de grano se ve afectada por la competencia ejercida por la otra especie. Asimismo, afirman que el rendimiento de semilla y el índice de cosecha de frijol se redujeron significativamente cuando fue sembrado asociado con maíz, debido principalmente a la competencia por insumos de cada especie. Por su parte, Díaz et al. (2012), trabajaron con frijol asociado con girasol, mencionan que la cantidad de luz interceptada por los cultivos en asociación está relacionada directamente con el área foliar e influye de manera significativa en el rendimiento de los cultivos. Lo anterior coincide con lo señalado con Delgado et al. (2015), quienes indican que existe una disminución en la producción y rendimiento de grano en frijol, posiblemente debido al sombreado ejercido por el maíz. Vélez et al. (2007) mencionan que en la asociación maíz-frijol, el rendimiento del frijol disminuye más del $40 \%$ y en maíz entre10 y $20 \%$.

Cuadro 5. Correlación entre el rendimiento de grano de maíz con el número de granos (NG), tamaño de grano (TG) y granos por mazorca (GM).

\begin{tabular}{lcc}
\hline $\begin{array}{l}\text { Componentes de } \\
\text { rendimiento }\end{array}$ & $\begin{array}{c}\text { Coeficiente de } \\
\text { determinación de } \mathrm{R}^{2}\end{array}$ & Probabilidad de F \\
\hline $\mathrm{NG}$ & 0.98 & $* *$ \\
$\mathrm{TG}$ & 0.09 & $\mathrm{NS}$ \\
$\mathrm{GM}$ & 0.01 & $\mathrm{NS}$ \\
\hline $\mathrm{NS}=$ no significativo $(P \leq 0.05) ; * *(P \leq 0.01)$. &
\end{tabular}




\section{Biomasa y Rendimiento en Grano y Rentabilidad de la Asociación Ayocote-Maíz}

Evaluando el frijol ayocote el tratamiento con mayor tasa de retorno (TR) fue el de 3 plantas de frijol ayocote con 3 de maíz (\$4.22), la tasa de retorno más baja fue el de 1 planta de frijol ayocote con 3 plantas de maíz (\$1.77) (Cuadro 6). Respecto al maíz el tratamiento con mayor TR fue con 3 plantas de maíz con 1 planta de frijol ayocote (\$5.66), el tratamiento con menor TR fue 1 planta de maíz con 1 planta de frijol ayocote (\$0.84) (Cuadro 7). Lo anterior se puede atribuir a que el incremento del número de plantas de maíz genera una mayor competencia interespecífica con las plantas de frijol ayocote disminuyendo el RG. Asimismo, los tratamientos con mayor número de plantas de frijol ayocote pueden desarrollar mayor cobertura del dosel que le permite captar mayor radiación solar, generando MS que se destina a la formación de vainas y al llenado de grano (Escalante y Rodríguez, 2011).

La BT y RG del agrosistema frijol ayocote-maíz mostró cambios significativos por el efecto del NPA y NPM por mata (Cuadro 8). Por otra parte en la asociación de ayocote con maíz azul, los tratamientos

Cuadro 6. Rendimiento (RG), Ingresos totales (IT), costos fijos (CF), costos variables (CV), costos totales (CT), ingresos netos (IN) y tasa de retorno (TR) de ayocote asociado con maíz chalqueño cv azul. Montecillo, México. Verano 2014.

\begin{tabular}{|c|c|c|c|c|c|c|c|c|}
\hline Tratamiento & BT & $\mathrm{RG}$ & IT & $\mathrm{CF}$ & $\mathrm{CV}$ & $\mathrm{CT}$ & IN & $\mathrm{TR}$ \\
\hline \multicolumn{7}{|c|}{$\ldots-\mathrm{gm}^{2} \ldots$} & \multicolumn{2}{|c|}{$\ldots-\ldots+\ldots$} \\
\hline $1 \mathrm{~A}+1 \mathrm{M}$ & 119 & 421 & 29750 & 5000 & 2127 & 7127 & 22623 & 3.17 \\
\hline $1 \mathrm{~A}+2 \mathrm{M}$ & 113 & 410 & 28250 & 5000 & 4115 & 9115 & 19135 & 2.09 \\
\hline $1 \mathrm{~A}+3 \mathrm{M}$ & 108 & 400 & 27000 & 5000 & 4725 & 9725 & 17275 & 1.77 \\
\hline $2 \mathrm{~A}+1 \mathrm{M}$ & 179 & 609 & 44750 & 5000 & 5761 & 10761 & 33989 & 3.15 \\
\hline $2 \mathrm{~A}+2 \mathrm{M}$ & 189 & 615 & 47250 & 5000 & 7072 & 12072 & 35178 & 2.91 \\
\hline $2 \mathrm{~A}+3 \mathrm{M}$ & 173 & 586 & 43250 & 5000 & 8979 & 13979 & 29271 & 2.09 \\
\hline $3 \mathrm{~A}+1 \mathrm{M}$ & 258 & 860 & 64500 & 5000 & 7334 & 12334 & 52166 & 4.22 \\
\hline $3 \mathrm{~A}+2 \mathrm{M}$ & 250 & 836 & 62500 & 5000 & 9091 & 14091 & 48409 & 3.43 \\
\hline $3 \mathrm{~A}+3 \mathrm{M}$ & 236 & 814 & 59000 & 5000 & 10651 & 15651 & 43349 & 2.76 \\
\hline
\end{tabular}

$\overline{\mathrm{A}}=$ frijol ayocote, $\mathrm{M}=$ maíz; el número indica el número de plantas. $\mathrm{BT}=$ biomasa total; (IT) Rendimiento por el costo de kg de frijol ayocote (\$25.00), (CF) Incluye costos de preparación del terreno, manejo de cultivo, manejo de maleza (mano de obra), manejo de plagas y enfermedades, transporte, cosecha; (CV) Precio y cantidad de semilla y fertilizante; (CT) Costo fijo + Costo variable; (IN\$) Ingreso total - Costo total; (TR \$) Tasa de retorno.

Cuadro 7. Rendimiento (RG), ingresos totales (IT), costos fijos (CF), costos variables (CV), costos totales (CT), ingresos netos (IN) y tasa de retorno (TR) de maíz chalqueño cv azul en asociación con frijol ayocote. Montecillo, México. Verano 2014.

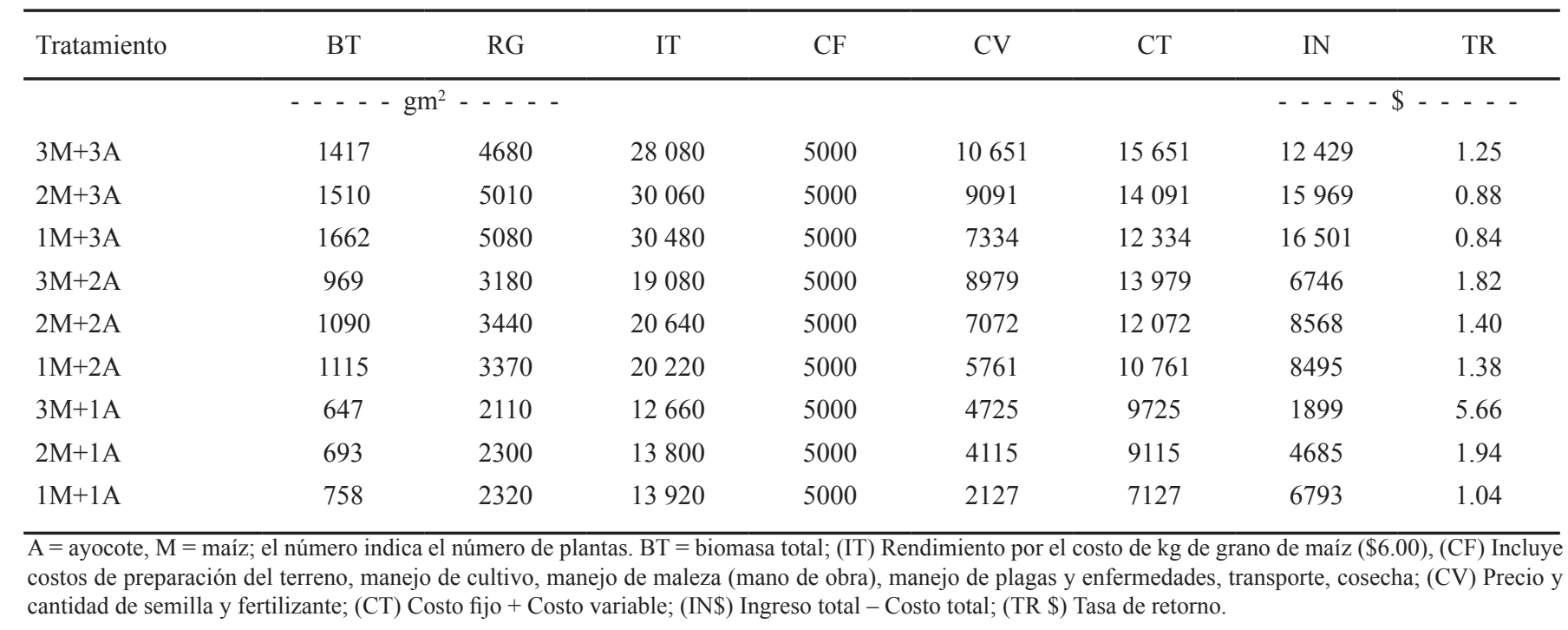


Cuadro 8. Rendimiento (RG), Ingresos totales (IT), costos fijos (CF), costos variables (CV), costos totales (CT), ingresos netos (IN) y tasa de retorno (TR) en la interacción de frijol ayocote asociado con maíz chalqueño cv azul. Montecillo México. Verano 2014.

\begin{tabular}{|c|c|c|c|c|c|c|c|}
\hline Tratamiento & $\mathrm{RG}$ & IT & $\mathrm{CF}$ & $\mathrm{CV}$ & $\mathrm{CT}$ & IN & TR \\
\hline & $\mathrm{gm}^{2}$ & & & & & \multicolumn{2}{|c|}{$\ldots \ldots+\ldots$} \\
\hline $3 \mathrm{~A}+3 \mathrm{M}$ & 704 & 17600 & 5000 & 8840 & 11840 & 5760 & $2.05 \mathrm{ef}$ \\
\hline $2 \mathrm{~A}+3 \mathrm{M}$ & 675 & 16875 & 5000 & 5250 & 9750 & 7125 & $1.36 \mathrm{~g}$ \\
\hline $1 \mathrm{~A}+3 \mathrm{M}$ & 616 & 15400 & 5000 & 2425 & 8150 & 7250 & $1.12 \mathrm{~g}$ \\
\hline $3 \mathrm{~A}+2 \mathrm{M}$ & 568 & 14200 & 5000 & 8470 & 11470 & 2730 & $4.20 \mathrm{c}$ \\
\hline $2 \mathrm{~A}+2 \mathrm{M}$ & 533 & 13325 & 5000 & 4850 & 9150 & 4175 & $2.19 \mathrm{e}$ \\
\hline $3 \mathrm{~A}+1 \mathrm{M}$ & 469 & 11725 & 5000 & 6137 & 10137 & 1588 & $6.38 \mathrm{a}$ \\
\hline $1 \mathrm{~A}+2 \mathrm{M}$ & 451 & 11275 & 5000 & 1775 & 6775 & 4500 & $1.50 \mathrm{f}$ \\
\hline $2 \mathrm{~A}+1 \mathrm{M}$ & 409 & 10225 & 5000 & 3250 & 8750 & 1475 & $5.93 \mathrm{~b}$ \\
\hline $1 \mathrm{~A}+1 \mathrm{M}$ & 351 & 8775 & 5000 & 1520 & 6520 & 2255 & $2.89 \mathrm{~d}$ \\
\hline
\end{tabular}

$\mathrm{A}=$ frijol ayocote, $\mathrm{M}=$ maíz; el número indica el número de plantas. $\mathrm{BT}$ = biomasa total; (IT) Rendimiento por el costo de kg de frijol ayocote (\$25.00), grano de maíz (\$6.00), (CF) Incluye costos de preparación del terreno, manejo de cultivo, manejo de maleza (mano de obra), manejo de plagas y enfermedades, transporte, cosecha; (CV) Precio y cantidad de semilla y fertilizante; (CT) Costo fijo + Costo variable; (IN\$) Ingreso total - Costo total; (TR \$) Tasa de retorno.

con mayor tasa de retorno (TR) fueron $3 \mathrm{~A}+1 \mathrm{M}(\$ 6.38)$ seguido por $2 \mathrm{~A}+1 \mathrm{M}(\$ 5.93)$, el tratamiento $1 \mathrm{~A}+3 \mathrm{M}$ tuvo el TR más bajo (\$1.12) (Cuadro 4).

Estos resultados indican que al incrementar la DP se puede obtener mayor BT y RG en el agrosistema frijol ayocote-maíz, coincidiendo con Yilmaz et al. (2008), que trabajaron las ventajas de la asociación maíz-frijol, dichos autores mencionan que se obtiene un mayor IN cuando se siembran altas DP. Sin embargo, la asociación de $3 \mathrm{~A}+1 \mathrm{M}$ permite una mejor expresión de las plantas de ayocote en la producción de BT y de $\mathrm{RG}$, esto al tener menor competencia con el maíz por insumos (agua, radiación solar y nutrimentos), lo cual permite un mayor IN, en contraste con los tratamientos de altas DP, las cuales presentan mayor competencia por dichos insumos y consecuencia en la reducción del rendimiento (Tsubo et al., 2003).

\section{CONCLUSIONES}

En el agrosistema frijol ayocote-maíz, la producción de biomasa, rendimiento de grano y sus componentes, son afectados por el número de plantas de frijol ayocote y de maíz por mata. La combinación para lograr el mayor rendimiento en grano, producción de biomasa e ingreso neto se logró con tres plantas de frijol ayocote y una de maíz. Tanto la biomasa como el rendimiento de las especies agrícolas involucradas en la asociación frijol ayocote-maíz, son afectados por los cambios en densidad de población por mata. La mayor tasa de retorno por peso invertido en la asociación se logra con la combinación de tres plantas de frijol ayocote con una de maíz.

\section{LITERATURA CITADA}

Alves, A. F., M. J. B. Andrade, N. M. B. Vieira, and P. M. Rezende. 2008. Grain yield of four new cultivars based on plant density. Ann. Report Bean Improv. Coop. 51: 242-243.

Clark, E. A. and C. A. Francis. 1985. Bean - maize intercrops: A comparison of bush and climbing bean growth habits. Field Crops Res. 10: 151-166.

Apáez B., P., J. A. S. Escalante-Estrada y M. T. RodríguezGonzález. 2013. Producción de vaina verde en frijol chino y tipo de espaldera en clima cálido. Rev. Chapingo Ser. Hortic. 19: 129-140.

Ayala-Garay, O. J., J. M. Pichardo-González, J. A. EstradaGómez, J. A. Carrillo-Salazar y A. Hernández-Livera. 2006. Rendimiento y calidad de semilla de frijol ayocote en el Valle de México. Agric. Téc. Méx. 32: 313-321.

Basurto, F., D. Martínez, A. Castellanos y M. A Martínez. 1996. Ciclo agrícola y fenología de Phaseolus coccineus L. en sistemas de agricultura tradicional en la Sierra Norte de Puebla, México. Etnoecológica 3: 71-82.

Barrios-Gómez, E. J., C. López-Castañeda, J. Kohashi-Shibata, J. A. Acosta-Gallegos, S. Miranda-Colín y N. Mayek-Pérez. 2010. Rendimiento de semilla, y sus componentes en frijol flor de mayo en el Centro de México. Agrociencia 44: 481-489.

Barrios-Gómez, E. J., C. López-Castañeda, J. A. Acosta-Gallegos, S. Miranda-Colín y J. Canul-Ku. 2011. Efecto del estrés hídrico en el crecimiento y desarrollo de frijol. Invest. Agropec. 8: 1-15.

Delgado M., R., J. A. S. Escalante-Estrada, R. Díaz R., A. Trinidad S., E. J. Morales R. y E. Sosa M. 2014. Defoliación en maíz y su efecto sobre el rendimiento de frijol-maíz en asociación. Rev. Mex. Cienc. Agríc. 5: 1015-1027. 
Delgado M., R., J. A. S. Escalante-Estrada, E. J. Morales, J. A. López y M. Rocandio. 2015. Producción y rentabilidad del frijol ejotero (Phaseolus vulgaris L.) asociado a maíz en función de la densidad y el nitrógeno en clima templado. Rev. FCA UNCUYO 47: 15-25.

Díaz López, E., J. A. S. Escalante-Estrada, M. T. RodríguezGonzález y A. Gaytán. 2010. Producción de frijol ejotero en función del tipo de espaldera. Rev. Chapingo Ser. Hortic. 16: 215-221.

Escalante-Estrada, J. A. S. 2001. Biomasa, rendimiento, eficiencia en el uso del agua y nitrógeno en girasol de humedad residual. Terra 19: 19-27.

Escalante-Estrada. J. A. S., M. T. Rodríguez-González y L. E. Escalante-Estrada. 2006. Beans (Phaseolus vulgaris L.) yield in relation to growth habit, plant density and nitrogen fertilization. Ann. Rep. Bean Improv. Coop. 49: 253-254.

Escalante-Estrada, J. A. S. y M. T. Rodríguez-González. 2010. Biomasa, índice de cosecha y componentes de rendimiento en frijol y nitrógeno. Rev. Cienc. Agrí. Infor. 19: 5-11.

Escalante-Estrada, J. A. S. y M. T. Rodríguez-González. 2011. Biomasa y rendimiento de haba en función de la densidad de población, nitrógeno y fósforo. Cienc. Agríc. Inf. 20: 16-25.

Escalante-Estrada, J. A. S., M. T. Rodríguez-González y Y. I. Escalante-Estrada. 2013. Aplicación dividida de nitrógeno, $\mathrm{su}$ efecto sobre la eficiencia agronómica, rendimiento y componentes en frijol. Cienc. Tecnol. Agrop. Méx. 1: 52-55.

Escalante-Estrada, J. A. S., M. T. Rodríguez-González y Y. I. Escalante-Estrada. 2014. Tasa de crecimiento de biomasa y rendimiento de frijol en función del nitrógeno. Cienc. Tecnol. Agrop. Méx. 2: 1-8.

Escalante-Estrada, J. A. S., M. T. Rodríguez-González y Y. I. Escalante-Estrada. 2015. Acumulación y distribución de materia seca en cultivares de maíz asociados con frijol en clima templado. Rev. Mex. Cienc. Agríc. 1: 139-143.

Escalante-Estrada, J. A. S. y J. Kohashi-Shibata. 2015. El rendimiento y crecimiento del frijol. Un manual para toma de datos. Colegio de Posgraduados. Montecillo, Texcoco, México.

Fageria, N. K., V. C. Baligar, A. Moreira, and T. A. Portes. 2010. Dry bean genotypes evaluation for growth, yield components and phosphorus use efficiency. J. Plant Nutr. 33: 2167-2181.

Galdámez-Galdámez, J., C. E. Aguilar-Jiménez, A. GutiérrezMartínez, J. A. Morales-Cabrera, S. Mendoza-Pérez y F. Martínez-Aguilar. 2010. Maíz asociado con frijol, canavalia (Canavalia ensiformis L.) y calabaza (Cucurbita moschata Duch) en Villaflores, Chiapas. Quehacer Cient. Chiapas 1: 18-29.

García, E. 2005. Modificaciones al sistema de clasificación climática de Köppen: Para adaptarlo a las condiciones de la República Mexicana. Instituto de Geografía UNAM. México, D. F.

Garduño G., J., E. J. Morales, S. Guadarrama V. y J. A. Escalante E. 2009. Biomasa y rendimiento de frijol con potencial ejotero en unicultivo y asociado con girasol. Rev. Chapingo Ser. Hortic. 15: 33-39.
Hanway, J. J. 1963. Growth stages of corn (Zea mays L.). Agron. J. 55: 487-492.

Jana A., C., P. Barriga B., A. Krarup H. y R. Fuentes P. 2000. Eficiencia de la asociación maíz (Zea mays) y frijol (Phaseolus vulgaris). Agro Sur 28: 71- 80.

Jiménez G., J. C. y J. A. Acosta G. 2013. Efecto de la densidad a simple y doble hilera en el rendimiento de frijol de temporal en Chihuahua. México. Rev. Mex. Cienc. Agríc. 4: 393-407.

Masaya, P. and J. W. White. 1991. Adaptation to photoperiod and temperature. pp. 445-500. In: A. van Schoonhoven and O. Voysest (eds.). Common beans: Research for crop improvement. C. A. B. Intl. U. K. and CIAT. Cali, Colombia.

Morgado, L. B. and R. W. Willey. 2008. Optimum plant population for maize-bean intercropping system in the Brazilian semi-arid región. Sci. Agric. (Piracicaba, Braz.) 65: 474-480.

Mutungamiri A., I. K. Mariga, and O. A. Chivinge. 2001. Effect of maize density, bean cultivar and bean spatial arrangement on intercrop performance. Afr. Crop Sci. J. 9: 487-497.

Osuna C., E. S., L. Reyes M., J. S. Padilla R. y M. A. Martínez G. 2012. Rendimiento de frijol Pinto Saltillo en altas densidades de población bajo temporal. Rev. Mex. Cienc. Agríc. 3: 1389-1400.

Ritchie, J. T. and D. S. NeSmith. 1991. Temperature and crop development. pp. 5-29. In: Hanks, J. and J. T. Ritchie (eds.). Modeling plant and soil systems. Agronomy Monograph no. 31. ASA, CSSA, and SSSA. Madison, WI, USA.

Rojas-Victoria, N. J., J. A. S. Escalante-Estrada, and M. T. Rodríguez-González. 2015. Biomass and yield of runner bean (Phaseolus coccineus L.) in association with maize. Ann. Rep. Bean. Improv. Coop. 58: 129-130.

Salinas R., N., J. A. S. Escalante-Estrada, M. T. Rodríguez-González y E. Sosa-Montes. 2008. Rendimiento y calidad nutrimental de frijol ejotero (Phaseolus vulgaris L.) en fechas de siembra. Rev. Fitotec. Mex. 31: 235-241.

Santalla, M., P. A. Casquero, and A. M. de Ron. 1999. Yield and yield components from intercropping bush vean cultivars with maize. J. Agron. Crop Sci. 183: 263-269.

Tsubo, M., E. Mukhala, H. O. Ogindo, and S. Walker. 2003. Productivity of maize-bean intercropping in semi-arid region of South Africa. Water 29: 381-388.

Tsubo, M. and S. Walker. 2004. Shade effects on Phaseolus vulgaris L. intercropped with Zea mays L. under well-watered conditions. J. Agron. CropSci. 190: 168-176.

Vélez V., L. D., J. Clavijo P., G. A. Ligarreto M. 2007. Análisis ecofisiológico del cultivo asociado maíz (Zea mays L.) - frijol voluble (Phaseolus vulgaris L.). Rev. Fac. Nal. Agr. Medellín 60: 3965-3984.

Volke, V. H. 1982. Optimización de insumos de la producción en la agricultura. Ed. Colegio de Postgraduados. Centro de Edafología. México.

Yilmaz, S., M. Atak, and M. Erayman. 2008. Identification of advantages of maize-legume intercropping over solitary cropping through competition indices in east mediterranean region. Turk. J. Agric. For. 32: 111-119. 